\title{
Electron Microscopic Observations of the Nucleus, Glial Dome, and Meninges of the Rat Acoustic Nerve'
}

\author{
MURIEL D. ROSS AND WILLIAM BURKEL \\ Department of Anatomy, The University of Michigan, \\ Ann Arbor, Michigan 48104
}

\begin{abstract}
The rat acoustic nerve is separated into central and peripheral portions by an astrocytic glial dome which is convex peripheralward. The long central portion is of typical central nervous system structure with narrow extracellular space (100-200 $\mathrm{A}$ in width), oligodendrocytes and astrocytes. The glial dome is penetrated by acoustic nerve fibers at a node of Ranvier; the basal lamina of the astrocytes is reflected back over the peripheral Schwann cells at this site. Centrally, the myelin is thinner than peripherally.

Acoustic nerve neurons, ranging in size from 25-60 $\mu$, occur in the central portion of the nerve and may be divided into two groups based upon size and density of organelles: large and medium-sized. All the neurons possess an eccentric nucleus and a peripheral clear zone in the perikaryon beneath which Nissl substance is aggregated, but the medium-sized neurons have fewer organelles than the large cells. Dendrites and axons are similar in ultrastructure.

While collagenous fibrils, fibroblasts, Schwann cells and extensive extracellular space occur in the peripheral portion of the nerve, no structure corresponding to perineurium or epineurium exists. Instead, dura mater surrounds the acoustic nerve within the modiolus and the pia mater encloses bundles of nerve fibers up to the modiolar foramina where it is reflected back as arachnoid mater. Nerve fibers traversing the modiolar foramina are devoid of a meningeal or perineurial covering; this condition also prevails in the osseous spiral lamina, although wisps of pia-like cells enclose groups of ganglion cells and nerve fibers in the spiral tract. These findings may help to explain acoustic nerve involvement in pathological processes such as meningitis and encephalitis.
\end{abstract}

Acoustic nerve neurons have been described by a series of investigators using different technical approaches but obtaining conflicting results. Tarlov ('37) considered them to be similar in type to cells of the central nervous system; he found the cells in the human acoustic nerve to be frequently multipolar and to lie central to the glial dome. Although he did not describe the cells further, a photomicrograph used in his article shows the neurons to possess an eccentric nucleus. Harrison et al. ('62) and Harrison and Warr ('62), on the basis of their silver studies, described neurons in the acoustic nerve of the rat and mouse which they named the "acoustic nerve nucleus." Their results indicated that the neurons were bipolar, with their axons projecting centralward into the re- gion of the trapezoid body. The cells were shown to receive many terminals from fibers of small diameter which appeared to arise as collaterals from acoustic nerve fibers. These terminals disappeared when the cochlea was destroyed. Thus, these observers considered the acoustic nerve neurons to be second order acoustic neurons. Ross ('69) located similar cells in the mouse and rat which, on the basis of histochemical results, were said to resemble autonomic neurons. Fluorescence studies indicated that the neurons were multipolar, had eccentric nuclei, and exhibited a weak yellowish-green fluorescence. Associated with some of the neurons was an orange

1 This paper was presented in part at the meeting of the American Association of Anatomists in Chicago, Illinois, April, 1970. Supported by USPHS grants NB07306 and NB-07473. 
fluorescence, which might indicate the presence of an amine in a distinctive storage form (Ross, '69).

The numerous questions raised by the fluorescence results could not be answered within the limitations imposed by the fluorescence technique or the level of resolution possible with the fluorescence microscope. The ultrastructure of the neurons had never been described, although this might give supporting evidence to one viewpoint or another and might lead to a better localization of the orange fluorescent material. With these aims in mind, the present work was begun.

As the investigation proceeded, it became obvious that delineation of the glial dome and a description of the arrangement of the meninges around the acoustic nerve fell within the scope of the investigation. These results are pertinent to anatomical localization of the acoustic nerve neurons and thus are included here.

\section{MATERIAL AND METHODS}

Material used in this investigation was obtained from albino rats weighing 40$200 \mathrm{gm}$. The animals were anesthetized by intraperitoneal injection of $3.5 \%$ chloral hydrate $(1 \mathrm{ml} / 100 \mathrm{gm}$ body weight). The tissue was fixed by vascular perfusion of a solution of glutaraldehyde-paraformaldehyde (Karnovsky, '65). After shaving, the ventral thoracic wall was removed quickly and a cannula made from an 18 gauge needle, through which the perfusate was already running, was inserted into the left ventricle. Immediately, the right atrium was sectioned to permit venous outflow. The perfusion was continued for about 15 minutes.

Upon completion of the perfusion, the skull was opened and the cerebral hemispheres removed. The eighth nerve, together with a small piece of the ventral cochlear nucleus, was carefully dissected out. In some specimens, the petrous portion of the temporal bone was broken away and part of the modiolus and spiral ganglion were removed with the nerve. In other cases, the entire cochlea was removed and placed in ethylenediamine tetraacetic acid (EDTA) glutaraldehyde (Baird et al., '67) for three weeks for decalcification.
All tissue was postfixed in $1 \% \mathrm{OsO}_{4}$ in $0.14 \mathrm{M}$ sodium cacodylate- $\mathrm{HCl}$ buffer to which $0.1 \% \mathrm{CaCl}$ had been added. After two hours, the tissue was washed in cacodylate buffer, dehydrated in alcohol followed by propylene oxide and embedded in Epon 812 (Luft, '61). After polymerization at $60^{\circ} \mathrm{C}$ for 48 hours, $1-2 \mu$ thick sections of the tissue blocks were made. The sections were stained at $80^{\circ} \mathrm{C}$ with $1 \%$ toluidine blue to which $1 \%$ borax had been added.

Using the toluidine blue-stained sections for orientation, tissue blocks were trimmed for ultramicrotomy. Thin sections were cut and mounted either unsupported on 200 mesh grids or on single hole grids covered with a Formvar membrane. The sections were stained with lead citrate (Reynolds, '63) and studied with Hitachi Hu 11A and RCA EMU $3 \mathrm{G}$ electron microscopes.

\section{OBSERVATIONS}

Neurons, ranging in size from 25-60 $\mu$, are arranged in linear fashion among the acoustic nerve fibers in the modiolus central to the glial dome. On the basis of their size and ultrastructure, the neurons may be classified into two overlapping categories: large, from 35-60 $\mu$; and mediumsized, from $25-35 \mu$ (figs. 1,2 ).

\section{Large acoustic nerve neurons}

Perikaryon. The large neurons (fig. 1) are ovoid or pear-shaped and seldom present more than one process in a given section. They possess an eccentric nucleus which is flattened on the side facing the bulk of the cytoplasm. Numerous indentations, involving both layers of the nuclear envelope, occur along the flattened surface. The chromatin is finely granular and tends to form small clumps in the nuclear sap. The nucleolus is eccentrically located.

The cytoplasmic organelles are highly organized, giving the cells a laminated appearance (figs. 1, 3). Immediately beneath the plasma membrane is a rim of cytoplasm, $1.5 \mu$ to $2.5 \mu$ thick, which appears rather clear. This clear zone may have been emphasized due to the prolonged decalcification, which might have leeched out ground substance. Within this zone are scattered polyribosomes, mitochondria and neurofilaments. 
Directly internal to the clear zone is a broken ring of patches of rough endoplasmic reticulum and free ribosomes (fig. 3 ), corresponding to the Nissl bodies of light microscopy (Palay and Palade, '55). The cisternae of the rough endoplasmic reticulum are branched and sinuous, but organized into four to six roughly parallel rows. Numerous polyribosomes are aggregated along the cisternae, giving the patches an exceedingly granular appearance. Smaller patches of rough endoplasmic reticulum, sometimes consisting of a single cistern which may be branched, and clumps of ribosomes occur deeper in the cytoplasm.

At the nuclear indentations, rough endoplasmic reticulum is organized into two to six laminae which lie parallel to the nuclear envelope. Polyribosomes are scattered along the outer lamina of the entire nuclear envelope.

Cisternae of the Golgi complex also are organized into patches which are dispersed in concentric, broken, C-shaped rings in the bulk of the cytoplasm (fig. 3). The cisternae do not appear to be specifically oriented in direction, but are commonly associated, or continuous with, the innermost cistern of a patch of rough endoplasmic reticulum. Patches of Golgi cisternae, oriented parallel to the nuclear envelope, sometimes encircle the nucleus, lying external to the laminae of roung endoplasmic reticulum at the site of the nuclear indentation.

Mitochondria are scattered in the cytoplasm, but are commonly situated at the periphery of a patch of Golgi complex or of rough endoplasmic reticulum (fig. 3 ). Many of the mitochondria are elongate with oblique or longitudinally oriented cristae.

Dense bodies of various sizes, which may correspond to lysosomes, occur in the neuroplasm. They are usually located near cisternae of the Golgi complex (fig. 3).

Cell processes. None of the processes of the large acoustic nerve neurons contain organized rough endoplasmic reticulum or abundant free ribosomes (figs. 1, $4,5)$. The bases of all the processes observed thus far are simple thickenings of the peripheral clear zone of the neuron. Mitochondria, which usually become more numerous at the base, are longitudinally oriented in the direction of the process (figs. 1, 4, 5). Microtubules are numerous in all the processes, but appear to lack conspicuous fasciculation. Thus dendrites and axons resemble one another ultrastructurally to a great extent.

Nevertheless, it has been possible to differentiate dendrites from the axon by the degree of branching of the processes and the number of terminals present. Processes which have a thick base (up to $20 \mu$ ), remain large (up to $10 \mu$ ), branch rapidly, and whose surface membranes are almost completely invested with terminal boutons, have been considered to be dendrites (fig. 4). Observations on processes considered to be axons are limited. The axon, in the few instances one has been sectioned optimally to show the hillock and initial portion of the process, generally has a smaller base (about $15 \mu$ ), a smaller diameter (4-5 $\mu$ ), and terminal boutons only at or close to the region considered to be the axon hillock (fig. 5). Glial processes envelop the axon more distally. None of the processes considered to be axons have been traced for more than $40 \mu$; over this distance the axon remains unbranched and unmyelinated.

Kinds and distribution of terminals. The numerous terminals which occur on the perikarya as well as on the dendrites of acoustic nerve neurons are of three structurally different types. One kind of terminal $\left(T_{1}\right)$ is a bulb-like or cup-like expansion occurring immediately distal to the last node of Ranvier of a thinly myelinated fiber. On one occasion, a favorable section showed a parent fiber branching and providing two end-bulbs along a dendrite. The terminal bouton of this type is densely populated with clear, round vesicles $400-800$ $\AA$ in diameter, an occasional vesicle with a dense core, and mitochondria. These terminals are most numerous at the bases of and along the dendrites (fig. 1,4).

A second type of terminal $\left(\mathrm{T}_{2}\right)$ is more prominently associated with the perikarya. These terminals make a broad contact with the cell surface, but are sparsely populated with vesicles $400-800 \mu$ in diameter and with mitochondria. They resemble the clear zone of the subjacent neuron in density (figs. 1, 4). The parent process appears to be broad and unmyelinated. 
The third type of terminal $\left(T_{3}\right)$ arises from a thinly myelinated axon; it is long and finger-like, and wraps around a portion of the cell membrane (figs. 2, 5). In one case, the terminal extended over 29 $\mu$ of the perikaryal surface, and several synaptic sites occurred.

All three types of terminals have been observed on a single cell. The areas intervening between terminals are filled with processes of astrocytes and oligodendroglia, unless a "satellite" cell, to be described below, is associated with the neuron.

Although definition of the ultimate origin of the fibers which terminate in these diverse ways on acoustic nerve neurons was beyond the scope of the present study, certain observations concerning the thinly myelinated fibers related to the neurons were made. These fibers are from $1-2.5 \mu$ in diameter and travel along capillaries in the islands of tissue related to the acoustic nerve neurons and their processes. The fibers are oriented almost without exception in a direction at right angles to the acoustic nerve fibers. In none of the sections investigated were collaterals observed to arise from acoustic nerve fibers and feed into the bundles of thinly myelinated axons running along the blood vessels.

\section{Medium-sized neurons}

The medium-sized neurons of the acoustic nerve nucleus are rounded or polygonal with eccentric nuclei (fig. 2). The nuclei correspond in both size and appearance to those of the larger neurons, the decreased volume of cytoplasm largely accounting for the difference in size. A peripheral clear zone is present. The rough endoplasmic reticulum is, in some cases, organized into large patches consisting of from five to seven laminae sparsely supplied with ribosomes. The Golgi complex does not occur in numerous patches, nor does it encircle the nucleus. One prominent mass of Golgi complex, consisting of concentric cisternae in an onion-like configuration, is sometimes conspicuous.

Medium-sized neurons have fewer synaptic boutons on their perikarya than do the large neurons of the acoustic nerve nucleus (figs. 1, 2). However, examples of each type of terminal occurring on the large cells have been observed on the medium-sized neurons.

\section{Associated cells}

Glial elements. Astrocytes and oligodendroglia (fig. 5), which are similar to those described previously by Maxwell and Kruger ('65) and Kruger and Maxwell ('66), occur in the central portion of the acoustic nerve. However, additionally there are two kinds of cells in this segment which differ to some extent from previously described glial elements. One type of cell occurs as a "satellite" to some of the acoustic nerve neurons, while the other is distributed irregularly among the nerve fibers of the root.

The "satellite" cells are polygonal (figs. 3,6 ), small in size (about $8 \mu$ in broadest diameter), with a proportionally large nucleus $(6 \mu)$. One side of the cell lies on the plasma membrane of an acoustic nerve neuron; another portion of the cell membrane is closely apposed to that of an oligodendrocyte. Tight junctions may occur between the "satellite" and the subjacent neuron; unfortunately, this cannot be stated with certainty, because in no case in which the cells are present have the entire membranes in contact been unbroken. The nucleus is drawn to the side of the cell resting on the neuron; the chromatin is coarsely clumped at the nuclear envelope, and a typical nucleolus is present. Large, but few, patches of rough endoplasmic reticulum with cisternae arranged in 4 to 6 parallel rows occur in the cell body and in some of its processes. In some sections, the Golgi complex is prominent (fig. 6); it is located in that portion of the cytoplasm above the nucleus. Associated with the Golgi complex, and scattered elsewhere free in the cytoplasm, are large vesicles with a dense core. These vesicles often appear to be oblong and range from 1600-1750 $\AA$ when measured in broadest diameter (fig. 6). Beneath the outer membrane is a lucid layer; the dense core is variable in size but usually measures 1300 $1400 \AA$ in diameter. Although the surfaces of the cells are indented in many places by various processes, no synaptic sites have been observed.

The second type of cell considered here may be a variation of an astrocyte (fig. 7). 
These cells are large, irregular in outline, and contain a round nucleus in which the chromatin is dense but dispersed. Large, but few, patches of rough endoplasmic reticulum occur in the cells; they consist of from five to seven laminae. Small patches of cisternae of the Golgi complex are of frequent occurrence, as are small aggregates of multivesicular bodies. Bundles of fibrils are present both near the nucleus and in areas distant from it. Small, round vesicles with dense cores occur in some of these cells. The vesicles range in diameter from $680-820 \AA$, while the dense core measures $270-410 \AA$ (fig. 7 ).

\section{Meninges}

For purposes of description we have found it convenient to divide the cochlear division of the eighth nerve into various segments. Thus, that part of the nerve extending from the ventral cochler nucleus to the glial dome is designated as the "central portion" of the nerve root. The segment from the glial dome to the foramina of the spiral tract is considered to be the "peripheral portion" of the cochlear nerve root. The next three segments, respectively, lie within the foramina of the spiral tract, within the spiral canal, and within the osseous spiral lamina.

Subpial astrocytes. The "central portion" of the cochlear nerve root displays ultrastructural features typical of the central nervous system. It extends well into the modiolus, but because of the dome-like nature of the transitional zone between central and peripheral portions, its exact extent varies. Throughout its distance, however, the central portion is enclosed in a continuous sheath of fibrous astrocytes (figs. 8, 9, 10). This astrocytic layer is smooth on its external surface. The internal surface is more irregular because the rounded nuclei of the astrocytes project into the neuropil and the astrocytic processes conform to the surfaces of the nerve fibers. The astrocytes themselves are similar to those of the neuropil except that they form a continuous sheet and are covered externally by a basal lamina. In some places this basal lamina is shared by an overlying pial cell, but frequently collagenous fibrils separate glia from pia. The adjacent cells making up the subpial astro- cytic sheet have interdigitating processes joined by zonulae or maculae occludentes.

The subpial layer continues along the nerve root until just before the nerve fibers begin to segregate into bundles and run toward the individual foramina of the spiral tract of the modiolus. At this point the astrocytic layer curves toward the center of the nerve, gradually transecting it. In longitudinal sections the astrocytes present an irregular arched profile with the concavity central and the convexity facing peripherally. In three dimensions the astrocytes form a glial dome across the nerve which delineates the peripheral extent of the central nervous system. The dome itself is not symmetrical, but begins more proximally on the side of the nerve corresponding to the basal turn of the modiolus. The peripheral surface of the glial dome is made up of many small astrocytic processes (fig. 8) and is indented by the cochlear nerve fibers as they penetrate it (fig. 9).

A basal lamina covers the peripheral surface of the astrocytes of the dome. This basal lamina reflects back to become continuous with that of the Schwann cells of the cochlear nerve fibers at the last peripheral internode (fig. 9). The indentations of the glial dome where cochlear nerve fibers pass through bear a structural resemblance to Virchow-Robin spaces around blood vessels. However, these spaces are not continuous with the subarachnoid space, but are internal to the pia and are part of a tissue space like the endoneural space of peripheral nerve.

Pia mater. The pia mater covering the cochlear nerve is continuous with and identical to that of the rest of the central nervous system (fig. 10). It consists of a thin continuous sheet of flattened cells separated from the subpial astrocytes by a basal lamina and in many places also by a layer of collagenous fibrils. In addition to covering the cochlear nerve itself, the pia forms a partition between the cochlear and vestibular divisions of the eighth nerve in areas where they are closely apposed. Where the subpial astrocytic layer transects the nerve to form the glial dome, the pia separates from the astrocytes and remains external to bundles of cochlear nerve fibers to form their only surface cov- 
ering (fig. 11). Thus, individual bundles, or several small bundles as a group are enclosed in sleeves of pia like the root sheaths of peripheral nerves (fig. 12). These sleeves continue from the origin of the bundles just distal to the glial dome to the point at which the bundles enter the bony foramina of the spiral tract (figs. 13, 14). Here, the pia doubles back to become continuous with the arachnoid, leaving the bundles of nerve fibers devoid of pial ensheathment as they traverse the foramina (fig. 15). Only within the spiral tract do cells resembling pia mater appear once more. In the latter location, the cells form wispy membranes which incompletely surround groups of ganglion cells, nerve fibers and capillaries. The pia-like cells are not found in the osseous spiral lamina, where the bundles of nerve fibers again lack typical fascicular sheaths.

Arachnoid mater. Except where they are continuous (fig. 14), the arachnoid is separated from the pia by a well defined subarachnoid space through which run many trabeculae and branches of the labyrinthine blood vessels. The arachnoid itself is composed of an electron-dense layer of cells similar to pia. It is closely apposed to the subjacent meningeal layer of dura mater and maculae adherentes are common between the two (fig. 16).

Dura mater. The dura lining the modiolus consists of two layers, the periosteal and the meningeal, which are separated from one another by a variable amount of tissue space (fig. 16). The periosteal layer is tightly adherent to the bone and is comprised of periosteal cells, overlying fibroblasts and interspersed collagenous fibrils. The periosteal cells throughout most of the modiolus are joined by maculae occludentes, forming a continuous sheet. Within the foramina of the spiral tract, however, this sheet is incomplete as the periosteal cells become scattered.

The meningeal layer of dura is closely apposed to the arachnoid mater within the modiolus, with only a 200-300 $\AA$ gap existing between the two. Hence, the interface between dura and arachnoid is difficult to determine and a subdural space appears to be more potential then real. The meningeal layer of dura is composed of several laminae of flattened, mesothelium-like cells which are separated from each other by a gap which usually does not exceed $200 \AA$. Maculae adherentes, and zonulae or maculae occludentes are found between adjacent cells without any apparent orientation.

In some areas, the periosteal and meningeal layers of dura are separated by only a few hundred angstroms, while elsewhere they lie as much as $100 \mu$ apart. Where the periosteal and meningeal layers are closely apposed, occasional junctional complexes are found. In areas where the two layers are widely separated, fibroblasts, capillaries and venous sinuses are abundant and form a loose mesh joining the two. In the latter case, the space between the cells and blood vessels contains a flocculent material.

About 100-200 $\mu$ proximal to the foramina of the spiral tract the meningeal and periosteal layers become closely apposed to form a single layer of dura. From this point to the foramina, the meningeal layer gradually decreases in thickness and, finally, disappears. At the openings of the foramina, the meninges are three cells thick, being composed of an arachnoid cell, a meningeal dural cell and a periosteal dural cell, all closely apposed. It is just beyond this point that the arachnoid is reflected back as pia mater and the dura ends. Consequently, the bundles of nerve fibers within the foramina of the spiral tract lack any continuous meningeal covering. Nor is any sheath comparable to epineurium or perineurium present. The nerve fibers and capillaries (when present) are simply embedded in a connective tissue matrix within the foramina. Vascular channels through the bone contain a similar ground substance, which surrounds the vessels.

\section{DISCUSSION}

The present findings essentially agree with Tarlov's ('37) original description of the acoustic nerve neurons; they are multipolar, have eccentric nuclei, and lie central to the glial dome. Multipolarity of the neurons is not readily apparent in single sections for the processes are few and the cell perikarya are large. Some neurons were examined in serial sections to demonstrate this. 
The most interesting finding concerning the neurons is that dendrites and axons resemble one another ultrastructurally. Processes categorized as axons were identifiable only in sections which showed them arising from the perikaryon and extending away from the cell over a short distance. In the few instances in which this ideal situation occurred, the process considered to be the axon was distinguished by its smooth, regular contour, lack of branching, and the lack of terminals ending upon it. Neither fasciculation of microtubules nor a dense undercoating occurred as described previously as always being present in the case of multipolar neurons of the central nervous system (Peters et al., '68; Palay et al., '68). The acoustic nerve neurons, then, either are exceptions to this rule or they are not of central nervous system type. In this context Osen ('69) failed to find any structure homologous to the acoustic nerve nucleus in her recent study of the ventral cochlear nucleus of the cat. Categories which might be considered for the neurons include displaced acoustic bipolar neurons, which the cells described here do not resemble in any way (Rosenbluth, '62), or autonomic neurons. Among the investigators who have previously mentioned the difficulty which exists in distinguishing the axon from the dendrites in postganglionic autonomic neurons at the light microscopic level is Botár ('66), and, at the level of the electron microscope, Elfvin ('63a,b) and Pick et al. ('64).

The site of orange fluorescence was not found to our satisfaction in the present material. Orange fluorescence is extremely labile in water and may not have been preserved under the conditions of this technique. Large, granulated vesicles are present in the "satellite" cells described here, but these cells occurred too infrequently to account for the quantity of orange fluorescence observed in histochemical studies.

"Satellite" cells do not appear to correspond to the previous descriptions of small cells found in the rat sympathetic ganglia which have been interpreted as being morphologically similar to both neurons and chromaffin cells (Grillo, '66) or as interneurons (Williams, '67; Williams and Palay, '69). However, they resemble closely the neuroglial cell type described by
Vaughn and Peters ('68) as being possibly neuroglial precursors or microglia. The cells containing multivesicular bodies and, occasionally, small granulated vesicles, appear to be astrocytes on the basis of their overall watery appearance and presence of patches of fibrils within them (Maxwell and Kruger, '65).

The ultrastructural features of the glial dome of the rat acoustic nerve are entirely similar to those of the monkey trigeminal sensory root as described by Maxwell et al. ('69). Distally, a sheath comparable to the perineurium of peripheral nerve is lacking on the acoustic nerve. Instead, pia mater ensleeves the bundles of acoustic nerve fibers which are approaching the foramina of the spiral tract and the entire nerve remains enclosed by dura mater up to the level of penetration of the modiolar bone by the bundles of nerve fibers. The pia is reflected back as arachnoid at the foramina, leaving the bundles devoid of fascicular ensheathment as they travel through the foramina toward the spiral canal. The reflection of pia as arachnoid suggests that the subarachnoid space does not communicate freely with the foramina of the spiral tract. Wisps of pia-like cells form incomplete sheaths around groups of spiral ganglion cells and nerve fibers, or around some of the capillaries, in the spiral canal but the bundles of nerve fibers in the osseous spiral lamina again lack fascicular ensheathment.

The absence of perineurium in the bony foramina of the cochlea is unlike the situation in other portions of the peripheral nervous system, including nerve fascicles in bone marrow (Shanthveerappa and Bourne, '66). This fact, together with the present findings concerning the glial dome and meninges, may be clinically significant if further work demonstrates similar conditions in the human, and may help to explain involvement of the acoustic nerve in encephalitis and meningitis.

\section{LITERATURE CITED}

Baird, I. L., W. B. Winborn and D. E. Bockman 1967 A technique of decalcification suited to electron microscopy of tissues closely associated with bone. Anat. Rec., 159: 281-289.

Botár, J. 1966 The Autonomic Nervous System: An Introduction to Its Physiological and Pathological Histology. Akadémiai Kiadó, Budapest. 
Elfvin, L. G. 1963a The ultrastructure of the superior cervical sympathetic ganglion of the cat. I. The structure of the ganglion cell processes as studied by serial sections. J. Ultrastruct. Res., 8: 403-440.

$1963 \mathrm{~b}$ The ultrastructure of the superior cervical sympathetic ganglion of the cat. II. The structure of the preganglionic end fibers and the synapses as studied by serial sections. J. Ultrastruct. Res., 8: 441-476.

Grillo, M. A. 1966 C. Electron microscopy of sympathetic tissues. Pharm. Rev., 18: 387-399.

Harrison, J. M., and W. B. Warr 1962 A study of the cochlear nuclei and ascending auditory pathway of the medulla. J. Comp. Neur., 119: 341-380.

Harrison, J. M., W. B. Warr and R. E. Irving 1962 Second order neurons in the acoustic nerve. Science, 138: 893-895.

Karnovsky, M. J. 1965 A formaldehyde-glutaraldehyde fixative of high osmolarity for use in electron microscopy. J. Cell Biol., 27: 137A138A.

Kruger, L., and D. S. Maxwell 1966 Electron microscopy of oligodendrocytes in normal rat cerebrum. Am. J. Anat., 118: 411-436.

Luft, J. H. 1961 Improvements in epoxy resin embedding methods. J. Biophys. Biochem. Cytol., 9: 409-414.

Maxwell, D. S., and L. Kruger 1965 The fine structure of astrocytes in the cerebral cortex and their response to focal injury produced by heavy ionizing particles. J. Cell Biol., 25: 141-157.

Maxwell, D. S., L. Kruger and A. Pineda 1969 The trigeminal nerve root with special reference to the central-peripheral transition zone: an electron microscopic study in the Macaque. Anat. Rec., 164: 113-126.
Osen, K. K. 1969 Cytoarchitecture of the cochlear nuclei in the cat. J. Comp. Neur., 136: 453-484.

Palay, S. L., and G. E. Palade 1955 The fine structure of neurons. J. Biophys. Biochem. Cytol., 1: 69-88.

Palay, S. L., C. Sotello, A. Peters and P. M. Orkand 1968 The axon hillock and the initial segment. J. Cell Biol., 38: 193-201.

Peters, A., C. C. Proskauer and I. R. KaisermanAbramof 1968 The small pyramidal neuron of the rat cerebral cortex. The axon hillock and initial segment. J. Cell Biol., 39: 604-619.

Pick, J., C. de Lemos and C. Gerdin 1964 The fine structure of sympathetic neurons in man. J. Comp. Neur., 122: 19-68.

Reynolds, E. S. 1963 The use of lead citrate at high $\mathrm{pH}$ as an electron opaque stain in electron microscopy. J. Cell Biol., 17: 208-212.

Rosenbluth, J. 1962 The fine structure of acoustic ganglia in the rat. J. Cell Biol., 12: 329-359.

Ross, M. D. 1969 Orange fluorescence in the acoustic nerve. J. Histochem. Cytochem., 17: 814-820.

Shanthaveerappa, T. R., and G. H. Bourne 1966 Perineural epithelium: a new concept of its role in the integrity of the peripheral nervous system. Science, 154: 1464-1467.

Tarlov, I. M. 1937 Structure of the nerve root. Arch. Neurol. Psychiat., 37: 555-583.

Vaughn, J. E., and A. Peters 1968 A third neuroglia cell type. An electron microscopic study. J. Comp. Neur., 133: 269-288.

Williams, T. H. 1967 Electron microscopic evidence for an autonomic interneuron. Nature, 214: $309-310$.

Williams, T. H., and S. L. Palay 1969 Ultrastructure of the small neurons in the superior cervical ganglion. Brain Res., 15: 17-34.

PLATE 1

EXPLANATION OF FIGURES

1 Large acoustic nerve neuron. The nucleus, which is eccentric, contains granular chromatin and an eccentric nucleolus. The perikaryon is typical and characterized by a peripheral, rather clear zone of cytoplasm beneath which the organelles are densely organized. Numerous terminal boutons which are either well-populated with vesicles $\left(T_{1}\right)$, or are broad and less vesiculated $\left(T_{2}\right)$, contact both the perikaryon and the dendrite which emerges from the cell at lower right (arrow). $\times 2400$.

2 Medium-sized acoustic nerve neuron. The peripheral clear zone is broad and the perikaryon is less densely occupied by organelles than are the larger neurons. This micrograph shows two finger-like terminals $\left(\mathrm{T}_{3}\right)$ wrapping around the perikaryon, making numerous synaptic contacts (arrows) $\times 4100$. 


\section{D. Ross and W. Burkel}

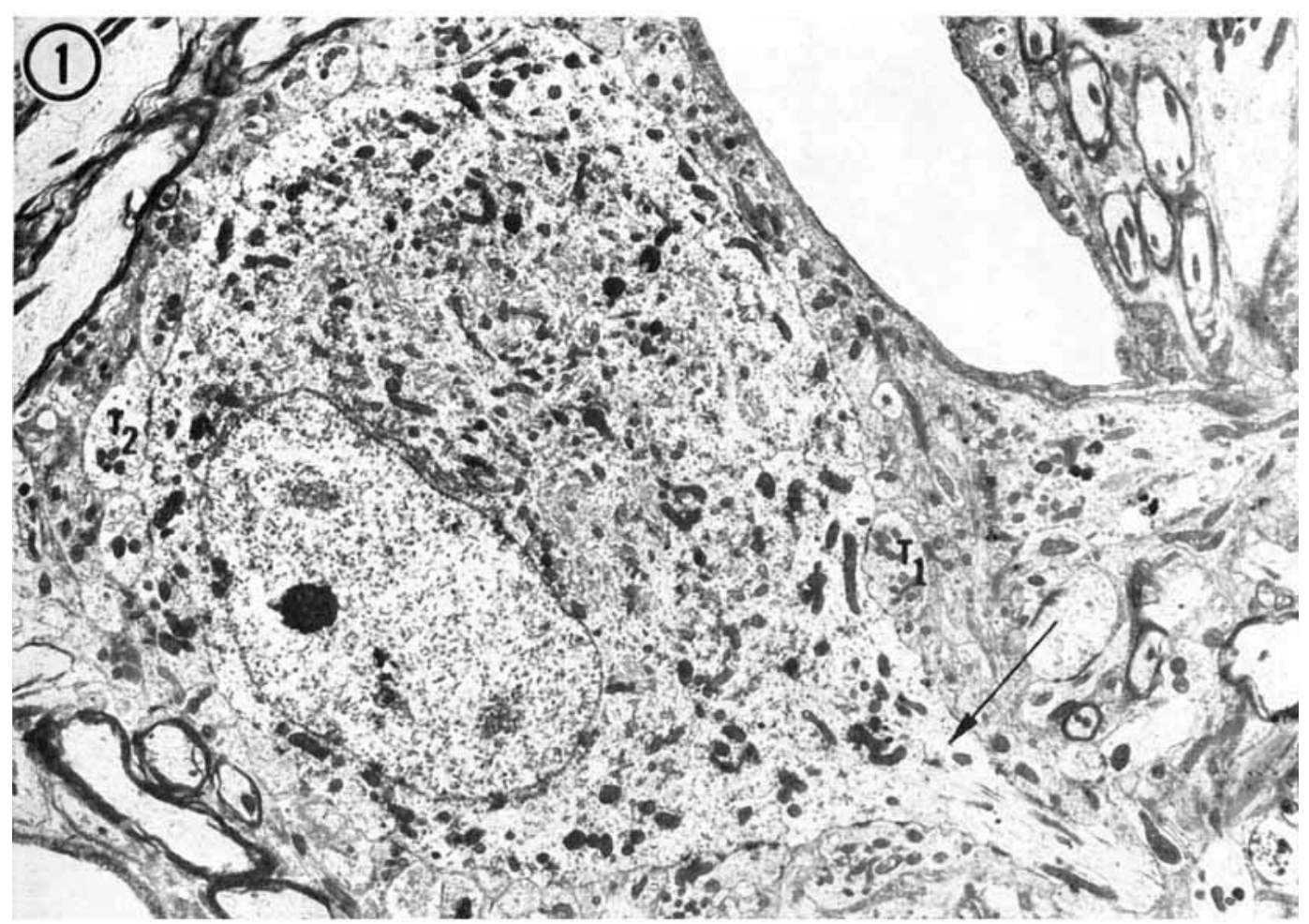

(2)

(2) (2)

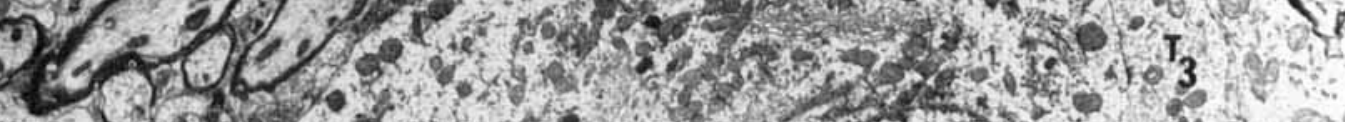

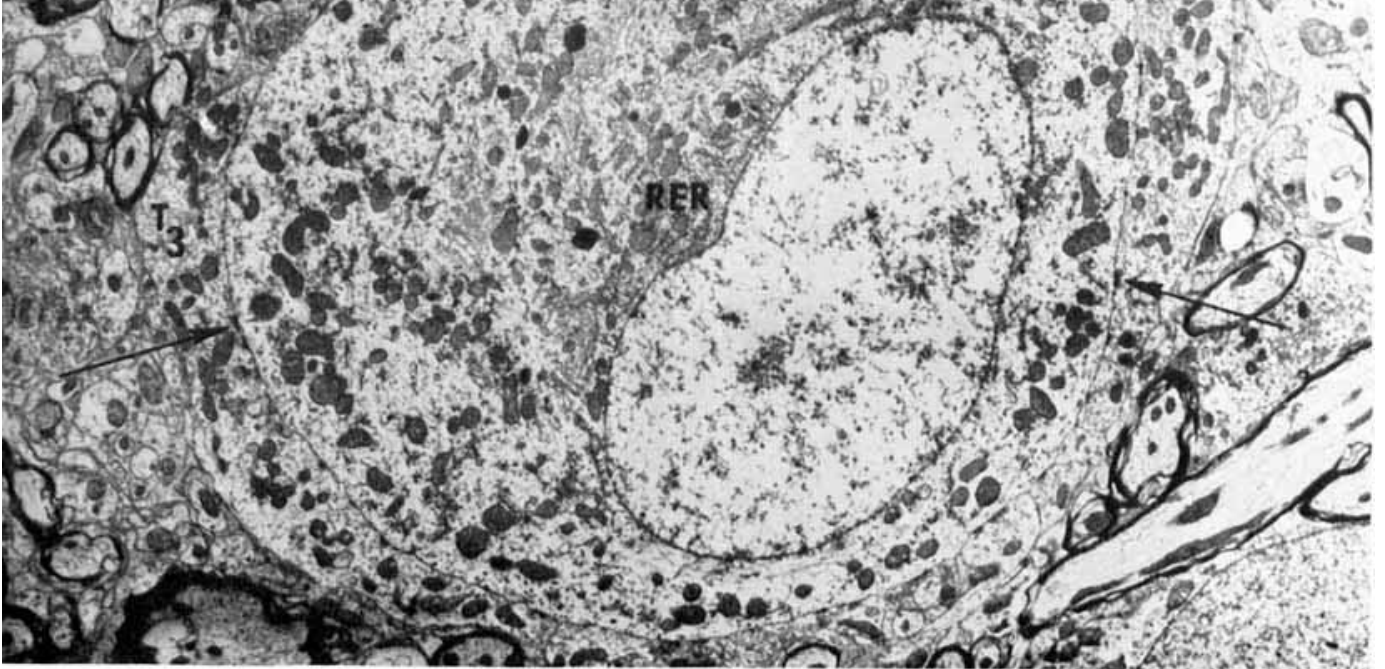




\section{PLATE 2}

EXPLANATION OF FIGURES

3 Large acoustic nerve neuron with "satellite" cell. The ultrastructural features of large acoustic nerve neurons are shown in greater detail here. $P$, peripheral clear zone; NB, organized rough endoplasmic reticulum corresponding to the Nissl bodies of light microscopy; G, Golgi complex; L, lysosome-like body. Note the small "satellite" cell, which is enlarged in figure 6, at the lower right (arrow). $\times 6400$.

4 Dendrite. This process, considered to be a dendrite, lacks organized rough endoplasmic reticulum or abundant free ribosomes, but contains numerous neurofilaments and microtubules. It is studded with terminal boutons $\left(T_{1}\right.$ and $\left.T_{2}\right) . \times 2700$.

5 Axon. This micrograph of a process considered to be an axon should be compared with figure 4 . While terminals $\left(T_{3}\right)$ occur in the region of the axon hillock ( $\mathrm{AXH}$ ), the more distal portion of this process is covered by astrocytic end-feet. No fasciculation of microtubules is apparent. A, astrocyte; O, oligodendrocyte. $\times 2800$. 

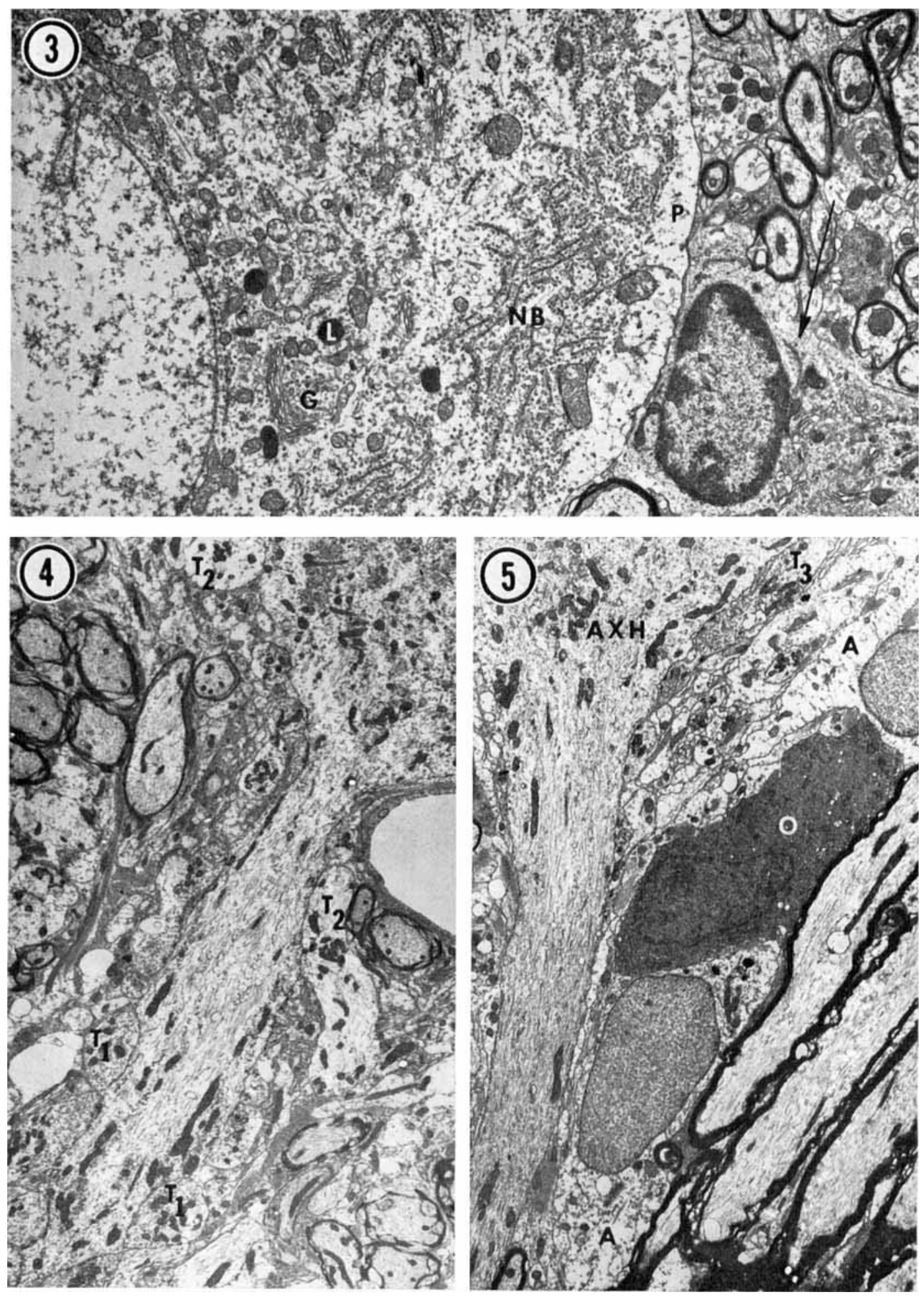

(5) 2013 - VAxH P

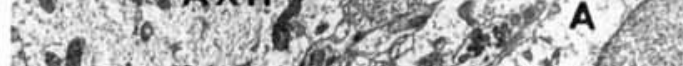

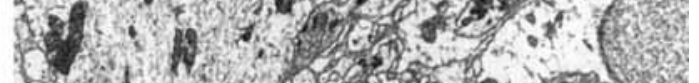

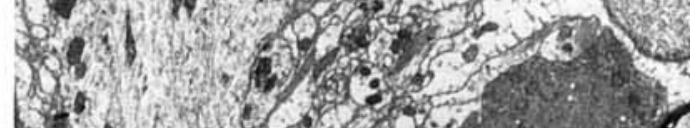
(1) 


\section{PLATE 3}

EXPLANATION OF FIGURES

6 "Satellite" cell. 'The "satellite" cell of figure 3 is shown in greater detail in this micrograph. The nucleus of the cell is basally disposed and the chromatin is clumped at the nuclear membrane. The Golgi complex (G) is prominent. A dense body, which may be a lysosome (L) is present in a process of the cell. Numerous granulated vesicles (arrows) occur in various parts of the cytoplasm. $\times 21,000$.

7 Astrocyte-like cell with granulated vesicles. This large, watery appearing cell which may be an astrocyte contains small, granulated vesicles. The vesicles (arrows) are shown in detail in the insert which is an enlargement of the region marked off by the rectangle. The cell contains a nucleus in which the chromatin material is granular and evenly disposed in the nucleoplasm, a bundle of fibrils (BF) near the nucleus, and laminated rough endoplasmic reticulum (RER). $\times$ 19,000 . Insert, $\times 34,000$. 
M. D. Ross and W. Burkel
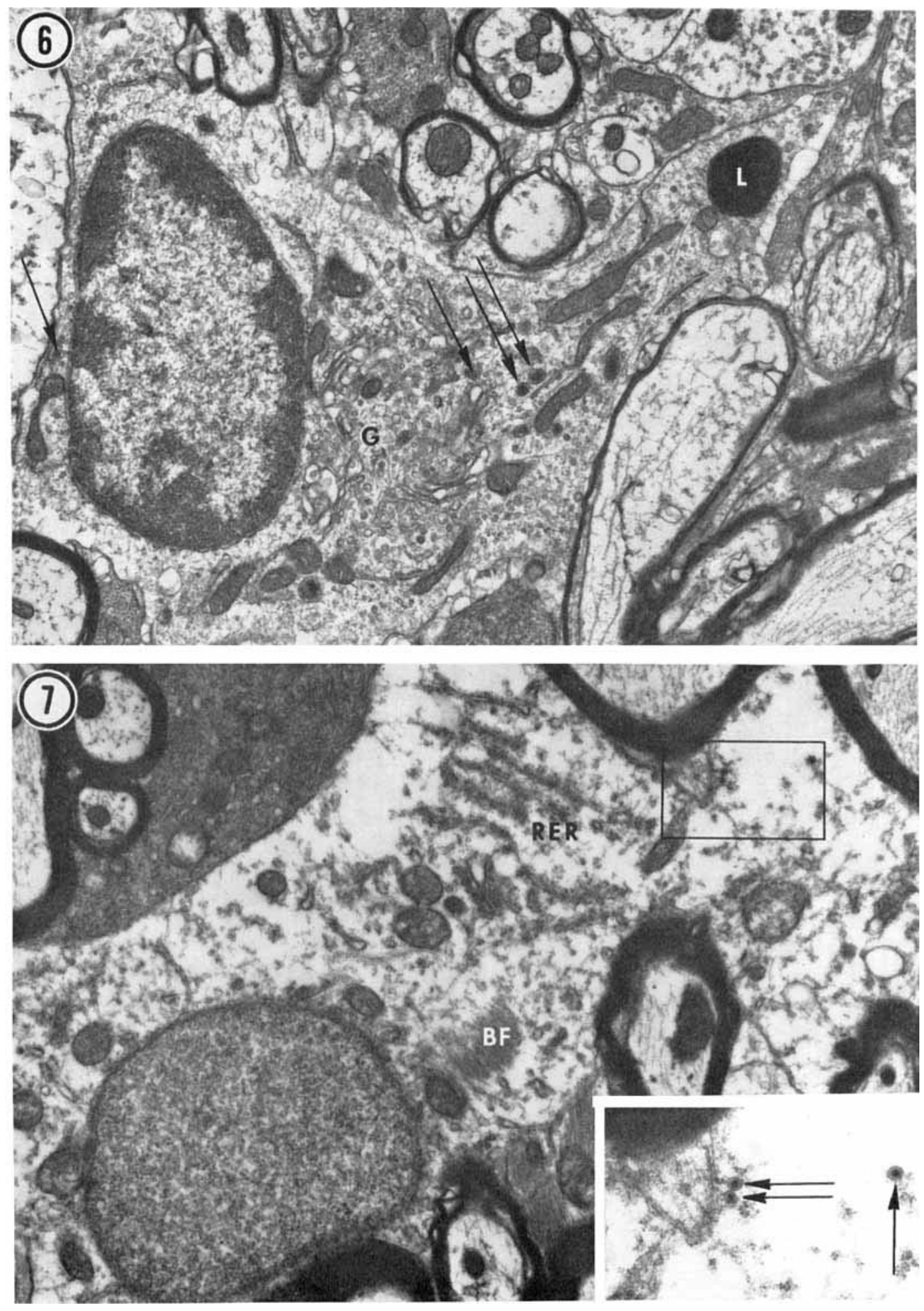
PLATE 4

EXPLANATION OF FIGURES

8 Glial dome. Interdigitating processes of fibrous astrocytes (A), joined focally by zonulae occludentes ( $\mathrm{ZO}$ ), make up the glial dome (GD). A basal lamina (BL) overlies the astrocytes, separating them from the tissue space (TSP) of the peripheral segment of the nerve. Two small astrocyte processes (AP), which project peripheralward from the dome out of the plane of this section, are seen above. $\times 35,000$.

9 Axon penetrating the glial dome. Where cochlear nerve fibers (AX) penetrate the glial dome (GD), the dome is indented so that there is a connective tissue space (TSP) around the axons similar to Virchow-Robin spaces around blood vessels. At the node of Ranvier (arrows) the basal lamina (BL) of the Schwann cell (SC) reflects back along the astrocytes (A), comprising the glial dome, $\times 11,000$. 
RAT ACOUSTIC NERVE

PLATE 4

M. D. Ross and W. Burkel
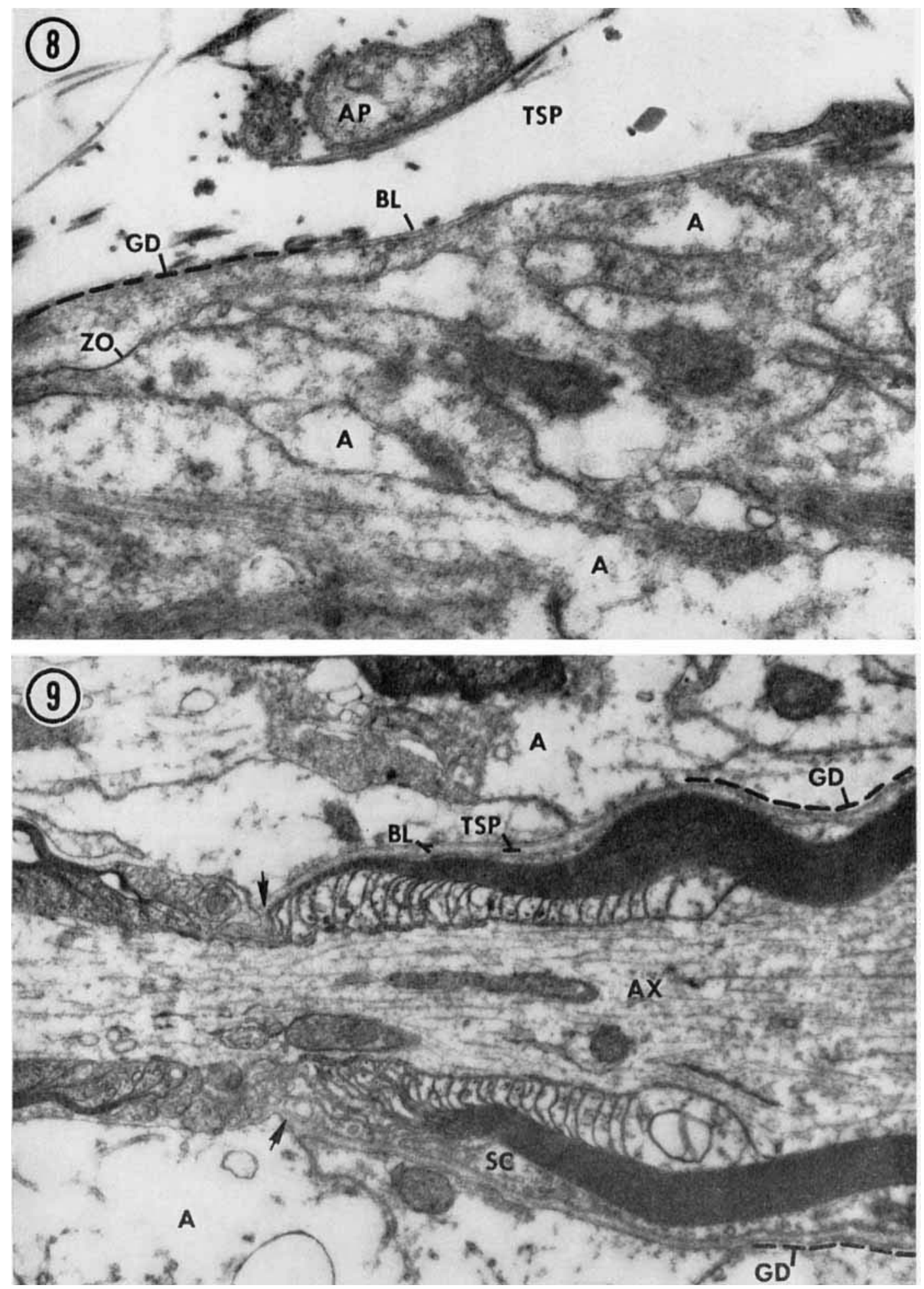
PLATE 5

EXPLANATION OF FIGURES

10 Central segment of cochlear nerve. Interdigitating processes of fibrous astrocytes (A) are covered by a basal lamina (BL). The thin, flattened pial cells (PM) separate the nerve proper from the subarachnoid space (SS). Collagenous fibrils (CO), running longitudinally with the nerve, separate astrocytes from pia. $\times$ 33,000 .

11 Peripheral segment of nerve. The nerve fibers of the peripheral segment are separated from the subarachnoid space (SS) only by a thin layer of pia (PM). Deep to the pial covering, this part of the cochlear nerve is similar to a peripheral nerve, having an extensive extracellular space (TSP) with fibroblasts (FB) and collagenous fibrils (CO). SC, Schwann cell. $\times 17,000$.

12 Peripheral bundle. Between the dome and the modiolar foramina the cochlear nerve fibers are gradually segregated into bundles of various sizes, each of which is surrounded by a sheath of pia (PM). SS, subarachnoid space; TSP, tissue space; CO collagenous fibrils. $\times 6700$.

13 Cochlear nerve near apex of the modiolus. Near the top of this micrograph peripheral bundles of nerves ( $P B$ ), surrounded by pia ( $P M$ ), are entering the modiolar foramina (FO). There is an unusually extensive subarachnoid space (SS) and extensive tissue space (TSP) between the meningeal (MD) and periosteai dura (PD) in this section. The tissue space contains many small blood vessels and dural sinuses (DS). At the arrow the pia covering the peripheral bundles and the arachnoid (AM) lining the modiolus become continuous. This area is enlarged in figure $14 . \times 120$.

14 Pia-arachnoid junction. At the point where peripheral bundles of cochlear nerve fibers enter the modiolar foramina the pia (PM) and arachnoid (AM) are continuous with one another (arrow). The meningeal (MD) and periosteal dura (PD) end at the pia-arachnoid turnback. Beyond this point only scattered perio. steal cells are found. In the peripheral segment of the nerve (to the left above) collagenous fibrils ( $\mathrm{CO}$ ) are abundant (see also figs. 11, 12). As the nerve bundles enter the bone (above right), collagenous fibrils become much finer (fig. 15). SS, subarachnoid space; B, bone. $\times 2,800$. 
RAT ACOUSTIC NERVE

PLATE 5

M. D. Ro:ss and W. Burkel
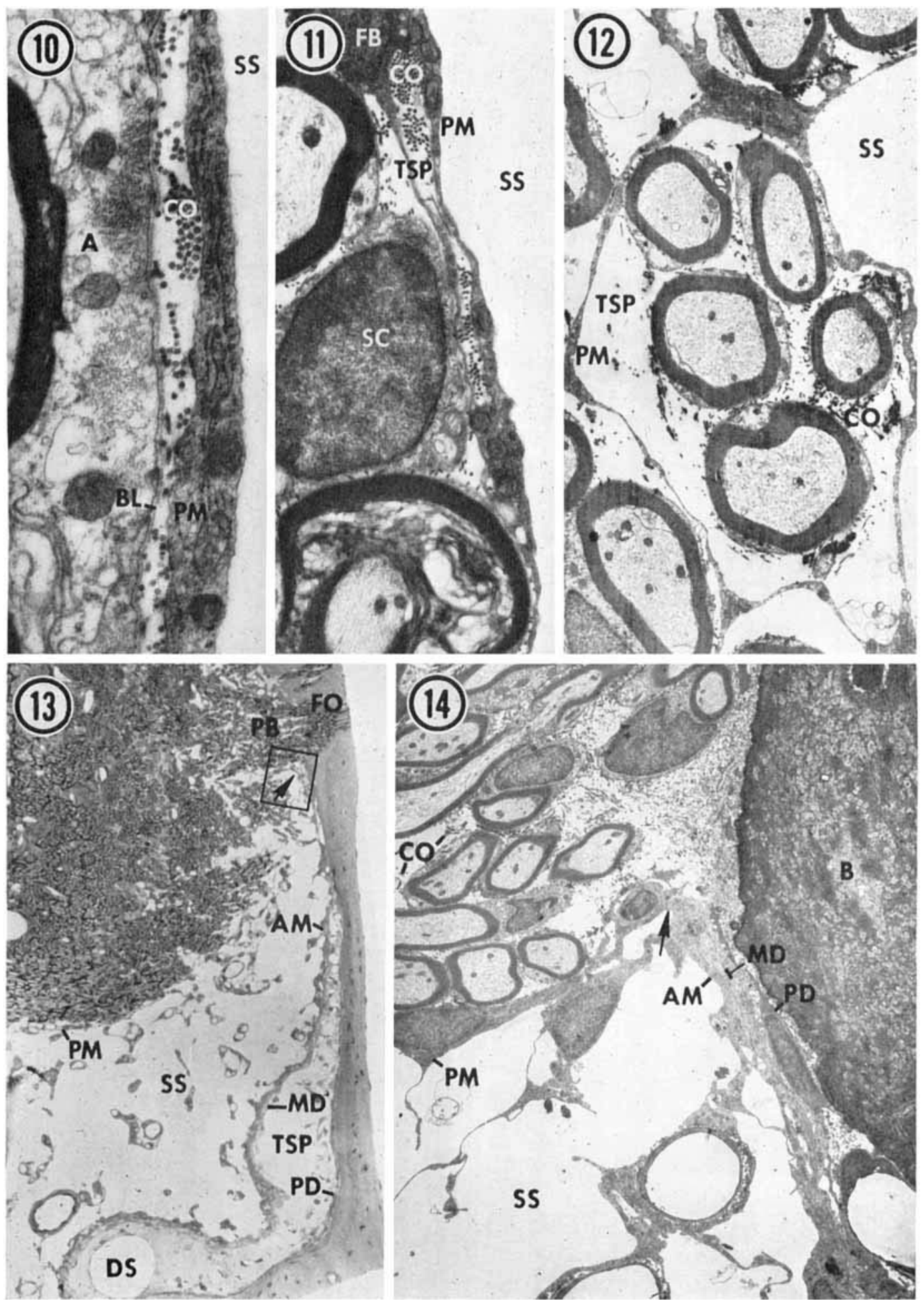
PLATE 6

EXPLANATION OF FIGURES

15 Cochlear nerve bundle in the modiolar foramen. Nerve bundles in the foramina are naked of any coverings comparable to meninges or peripheral nerve sheaths, such as perineurium. Unmyelinated nerves (UN) are common in these bundles. $\mathrm{PO}$, periosteal cell; $\mathrm{B}$, bone; $\mathrm{CO}$, collagenous fibrils. $\times 11,000$.

16 Arachnoid and dura. The modiolar bone (B) is lined by dura and arachnoid (AM) which are closely apposed to one another and attached by maculae adherentes (MA). The dura consists of a meningeal portion (MD), a connective tissue space (TSP) and a periosteal portion (PD). The tissue space typically contains a flocculent material. SS, subarachnoid space. $\times 15,000$. 

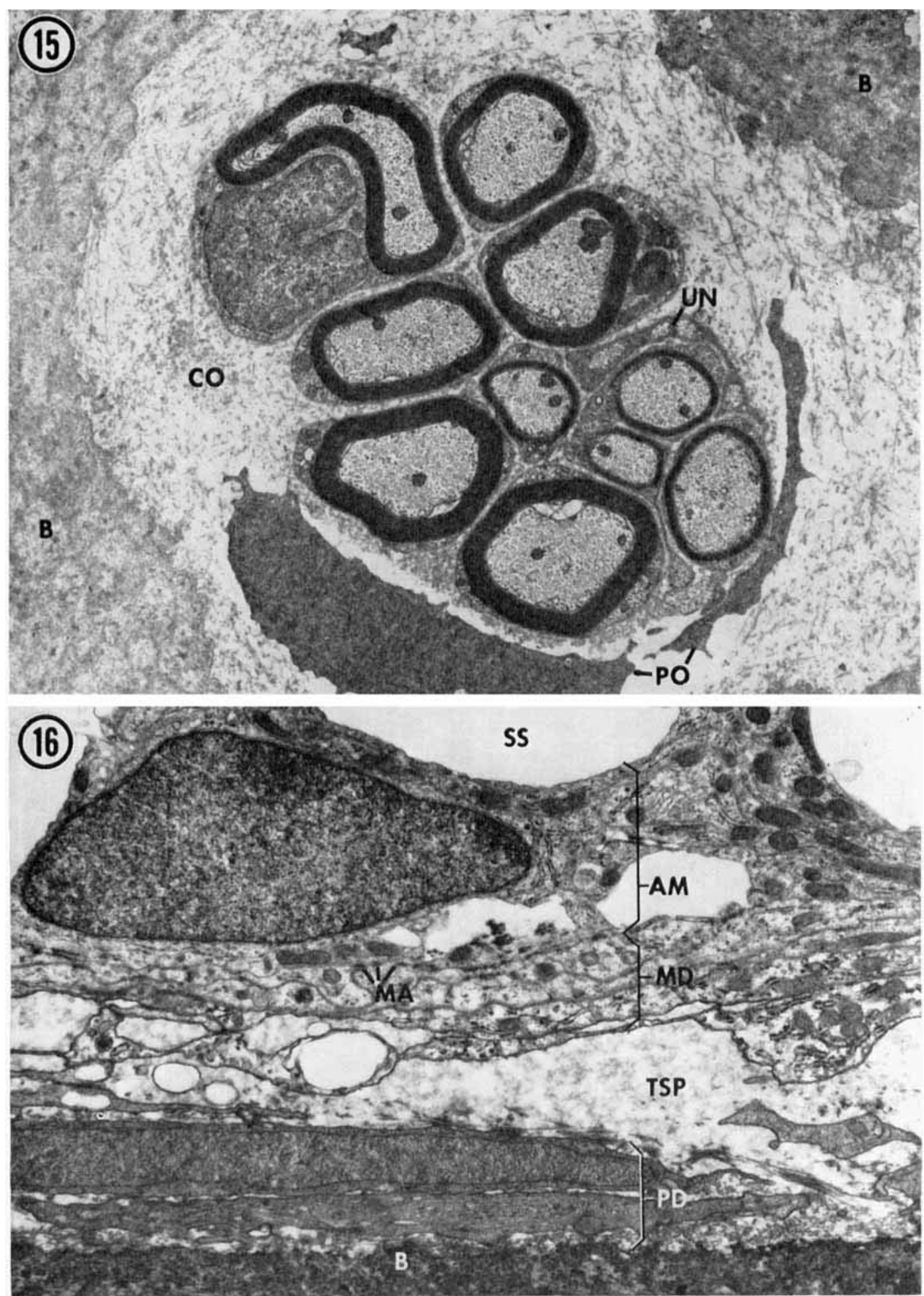